\title{
Existence of periodic solutions for a prescribed mean curvature Liénard $p$-Laplacian equation with two delays
}

\section{Zhiyan $\mathrm{Li}^{1,2^{*}}$, Tianqing $A n^{1}$ and Weigao $\mathrm{Ge}^{3}$}

"Correspondence: yylin79@126.com ${ }^{1}$ College of Science, Hohai University, Nanjing, 210098, China ${ }^{2}$ Department of Mathematics and Physics, Hohai University,

Changzhou Campus, Changzhou, 213022, China

Full list of author information is available at the end of the article

\begin{abstract}
This paper is concerned with the prescribed mean curvature Liénard type $p$-Laplacian equation with two arguments. By employing Mawhin's coincidence degree theorem and the analysis techniques, some new existence results of periodic solutions are obtained. We also give an example to illustrate the application of our main results.
\end{abstract}

Keywords: periodic solution; Mawhin's continuation theorem; Liénard type p-Laplacian equation

\section{Introduction}

Recently, the study of the periodic solutions of prescribed mean curvature equations has become very active; see [1-5] and the references therein. Meanwhile, the Liénard equation, Liénard system, and $p$-Laplacian equations are also studied by many people; see for example [6-8]. These kinds of equations have wide applications in many fields, such as physics, mechanics, and engineering.

In [9] Wang studied the following prescribed mean curvature Rayleigh equation with one deviating argument:

$$
\left(\varphi_{p}\left(\frac{x^{\prime}(t)}{\sqrt{1+x^{\prime 2}(t)}}\right)\right)^{\prime}+f\left(t, x^{\prime}(t)\right)+g(t, x(t-\tau(t)))=e(t)
$$

under the assumptions:

$$
\begin{aligned}
& f(t, u) \geq a|u|^{r}, \quad \forall(t, u) \in R^{2} \quad \text { and } \\
& g(t, u)-e(t) \geq-m_{1}|u|-m_{2}, \quad \forall t \in R, x \geq d .
\end{aligned}
$$

By using the theory of coincidence degree, the author obtained some sufficient conditions for the existence and uniqueness of periodic solution in the case of $\tau(t)=0$.

Stimulated by [9], we study the periodic solutions for a kind of prescribed mean curvature Liénard $p$-Laplacian equation with two deviating arguments in the form

$$
\left(\varphi_{p}\left(\frac{x^{\prime}(t)}{\sqrt{1+x^{\prime 2}(t)}}\right)\right)^{\prime}+f(x(t)) x^{\prime}(t)+g(x(t-\tau(t)))+h(x(t-\gamma(t)))=e(t),
$$

\section{Springer}

@2014 Li et al.; licensee Springer. This is an Open Access article distributed under the terms of the Creative Commons Attribution License (http://creativecommons.org/licenses/by/2.0), which permits unrestricted use, distribution, and reproduction in any medium, provided the original work is properly cited. 
where $\varphi_{p}(s)=|s|^{p-2} s, p \geq 2, \tau, \gamma$ are continuous functions with period $T, \tau^{\prime}(t)<1, \gamma^{\prime}(t)<1$, and $\int_{0}^{T} e(t) d t=0, f, g, h \in C^{1}(R, R)$.

To the best of our knowledge, there are few results on this topic. The purpose of this paper is to establish a criterion to guarantee the existence of $T$-periodic solution. Our methods and results are different from the corresponding ones of [9]. So our results are essentially new.

\section{Preliminaries}

Let $X$ and $Y$ be real Banach spaces and $L: D(L) \subset X \rightarrow Y$ be a Fredholm operator with index zero, that is, $X=\operatorname{Ker} L \oplus X_{1}$ and $Y=\operatorname{Im} L \oplus Y_{1}$. Furthermore, let $P: X \rightarrow \operatorname{Ker} L$ and $Q: Y \rightarrow Y_{1}$ be the continuous projectors. Clearly, $\operatorname{Ker} L \cap\left(D(L) \cap X_{1}\right)=\{0\}$, thus the restriction $L_{P}=\left.L\right|_{D(L) \cap X_{1}}$ is invertible. Denote by $K$ the inverse of $L_{P}$.

Let $\Omega$ be an open bounded subset of $X$ with $D(L) \cap \Omega \neq \Phi$. A map $N: \bar{\Omega} \rightarrow Y$ is called $L$-compact in $\bar{\Omega}$ if $Q N(\bar{\Omega})$ is bounded and the operator $K(I-Q) N: \bar{\Omega} \rightarrow X$ is compact. The following, Mawhin's continuation theorem, is well known.

Lemma 2.1 (Gaines and Mawhin [10]) Suppose that $X$ and $Y$ are two Banach spaces, and $L: D(L) \subset X \rightarrow Y$ is a Fredholm operator with index zero. Furthermore, $\Omega \subset X$ is an open bounded subset and $N: \bar{\Omega} \rightarrow Y$ is L-compact in $\bar{\Omega}$. If all the following conditions hold:

(1) $L x \neq \lambda N x, \forall x \in \partial \Omega \cap D(L), \lambda \in(0,1)$;

(2) $N x \notin \operatorname{Im} L, \forall x \in \partial \Omega \cap \operatorname{Ker} L$;

(3) $\operatorname{deg}\{J Q N, \Omega \cap \operatorname{Ker} L, 0\} \neq 0$, where $J: \operatorname{Im} Q \rightarrow \operatorname{Ker} L$ is an isomorphism.

Then the equation $L x=N x$ has a solution in $\bar{\Omega} \cap D(L)$.

To use Mawhin's continuation theorem to study (1.3), we firstly transform (1.3) into the following form:

$$
\left\{\begin{array}{l}
x_{1}^{\prime}(t)=\frac{\varphi_{q}\left(x_{2}(t)\right)}{\sqrt{1-\varphi_{q}^{2}\left(x_{2}(t)\right)}}, \\
x_{2}^{\prime}(t)=-f\left(x_{1}(t)\right) \frac{\varphi_{q}\left(x_{2}(t)\right)}{\sqrt{1-\varphi_{q}^{2}\left(x_{2}(t)\right)}}-g\left(x_{1}(t-\tau(t))\right)-h\left(x_{1}(t-\gamma(t))\right)+e(t) .
\end{array}\right.
$$

Denote by $\phi(x)=\frac{\varphi_{q}(x)}{\sqrt{1-\varphi_{q}^{2}(x)}}, \varphi_{q}(s)=|s|^{q-2} s, 1 / p+1 / q=1$, then prescribed mean curvature $p$-Laplacian operator $\varphi_{p}\left(\frac{x}{\sqrt{1+x^{2}}}\right)$ is marked by $\phi^{-1}(x)$. Clearly, if $x(t)=\left(x_{1}(t), x_{2}(t)\right)^{\top}$ is a $T$-periodic solution to (2.1), then $x_{1}(t)$ must be a $T$-periodic solution to (1.3).

Set $C_{T}^{1}=\left\{x: x \in C\left(R, R^{2}\right), x(t+T) \equiv x(t)\right\}$ with the norm $|x|_{0}=\max _{t \in[0, T]}|x(t)|, X=Y=$ $\left\{x(t)=\left(x_{1}(t), x_{2}(t)\right)^{\top} \in C\left(R, R^{2}\right): x(t+T) \equiv x(t)\right\}$ with the norm $\|x\|=\max \left\{\left|x_{1}\right|_{0},\left|x_{2}\right|_{0}\right\}$, $|x|_{2}=\left(\int_{0}^{T} x^{2}(t) d t\right)^{1 / 2}$. Then $X$ and $Y$ are Banach spaces. Let

$$
\begin{aligned}
& L: D(L) \subset X \rightarrow Y, \quad L x=x^{\prime}=\left(\begin{array}{l}
x_{1}^{\prime} \\
x_{2}^{\prime}
\end{array}\right), \\
& N: X \rightarrow Y, \\
& \quad \text { where } N x=\left(\begin{array}{c}
\phi\left(x_{2}(t)\right) \\
-f\left(x_{1}(t)\right) \phi\left(x_{2}(t)\right)-g\left(x_{1}(t-\tau(t))\right)-h\left(x_{1}(t-\gamma(t))\right)+e(t)
\end{array}\right) .
\end{aligned}
$$


It is easy to see that $\operatorname{Ker} L=R^{2}, \operatorname{Im} L=\left\{x: x \in Y, \int_{0}^{T} x(s) d s=0\right\}$. So $L$ is a Fredholm operator with index zero. Define projectors $P: X \rightarrow \operatorname{Ker} L$ and $Q: Y \rightarrow \operatorname{Im} Q$ by

$$
P x=\frac{1}{T} \int_{0}^{T} x(s) d s, \quad Q y=\frac{1}{T} \int_{0}^{T} y(s) d s,
$$

and let $K$ be the inverse of $\left.L\right|_{\operatorname{Ker} P \cap D(L)}$. Obviously $\operatorname{Ker} L=\operatorname{Im} Q=R^{2}$,

$$
[K y]^{-1}(t)=\int_{0}^{T} k(t, s) y(s) d s
$$

where

$$
k(t, s)= \begin{cases}\frac{s}{T}, & 0 \leq s<t \leq T \\ \frac{s-T}{T}, & 0 \leq t \leq s \leq T .\end{cases}
$$

From (2.3) and (2.4), one can easily see that $N$ is $L$-compact on $\Omega$, where $\Omega$ is an open bounded subset of $X$. The following lemma is useful to estimate $a$ priori bounds of periodic solutions of (2.1).

Lemma 2.2 (Liu et al. [11]) Suppose that $x(t) \in C_{T}^{1}$, and that there is a point $t_{*} \in R$ such that $x\left(t_{*}\right)=0$, then

$$
\int_{0}^{T}|x(t)|^{2} d t \leq \frac{T^{2}}{\pi^{2}} \int_{0}^{T}\left|x^{\prime}(t)\right|^{2} d t .
$$

Lemma $2.3([12])$ Let $\tau \in C_{T}^{1},\left|\tau^{\prime}\right|_{0}<1$. If $x(t) \in C_{T}^{1}$ then

$$
\int_{0}^{T}|x(t-\tau(t))|^{2} d t \leq \tau_{0} \int_{0}^{T}|x(t)|^{2} d t
$$

where $\tau_{0}=\frac{1}{1-\left|\tau^{\prime}\right|_{0}}, \gamma(t)$ satisfies the above inequality and $\gamma_{0}=\frac{1}{1-\left|\gamma^{\prime}\right|_{0}}$.

At the end of this section, we list the basic assumptions which will be used in Section 3.

$\left(\mathrm{H}_{1}\right)\left(g\left(x_{1}\right)-g\left(x_{2}\right)\right)\left(x_{1}-x_{2}\right)>0, x_{1}, x_{2} \in R$ and $x_{1} \neq x_{2},\left(h\left(x_{1}\right)-h\left(x_{2}\right)\right)\left(x_{1}-x_{2}\right)>0, x_{1}, x_{2} \in R$ and $x_{1} \neq x_{2}$.

$\left(\mathrm{H}_{2}\right)$ There is a constant $d>0$ such that $x(g(x)+h(x))>0$ if and only if $|x|>d$.

$\left(\mathrm{H}_{3}\right)$ There exist nonnegative constants $k_{1}, k_{2}, c_{1}, c_{2}$ such that

$$
\begin{array}{ll}
|g(x)| \leq k_{1}|x|+c_{1}, & |x|>d ; \\
|h(x)| \leq k_{2}|x|+c_{2}, & |x|>d .
\end{array}
$$

$\left(\mathrm{H}_{4}\right)$ There is a constant $\sigma>0$ such that $|f(s)| \geq \sigma$ for all $s \in R$.

\section{Main results and the proof}

In this section we state the main results and give its proof.

Theorem 3.1 Suppose the assumptions $\left(\mathrm{H}_{1}\right)-\left(\mathrm{H}_{4}\right)$ hold. Then (1.3) has at least one T-periodic solution provided $\frac{T}{\pi}\left(k_{1} \sqrt{\tau_{0}}+k_{2} \sqrt{\gamma_{0}}\right)<\sigma$. 
Proof Let $\Omega_{1}=\{x \in X: L x=\lambda N x, \lambda \in(0,1)\}$, suppose $x(t) \in \Omega_{1}$, then

$$
\left\{\begin{array}{l}
x_{1}^{\prime}(t)=\lambda \frac{\varphi_{q}\left(x_{2}(t)\right)}{\sqrt{1-\varphi_{q}^{2}\left(x_{2}(t)\right)}} \triangleq \lambda \phi\left(x_{2}(t)\right), \\
x_{2}^{\prime}(t)=-\lambda f\left(x_{1}(t)\right) \frac{\varphi_{q}\left(x_{2}(t)\right)}{\sqrt{1-\varphi_{q}^{2}\left(x_{2}(t)\right)}}-\lambda g\left(x_{1}(t-\tau(t))\right)-\lambda h\left(x_{1}(t-\gamma(t))\right)+\lambda e(t) .
\end{array}\right.
$$

Integrating both sides of the second equation of (3.1) from 0 to $T$, we have

$$
\int_{0}^{T}\left(g\left(x_{1}(t-\tau(t))\right)+h\left(x_{1}(t-\gamma(t))\right)\right) d t=0
$$

which implies that there exists a point $\xi \in[0, T]$ such that

$$
g\left(x_{1}(\xi-\tau(\xi))\right)+h\left(x_{1}(\xi-\gamma(\xi))\right)=0 .
$$

Now we claim that there must exist a point $t_{0} \in R$ such that

$$
\left|x_{1}\left(t_{0}\right)\right| \leq d
$$

In fact, let $\xi-\tau(\xi)=t_{1}, \xi-\gamma(\xi)=t_{2}$, then from (3.2), $g\left(x_{1}\left(t_{1}\right)\right)+h\left(x_{1}\left(t_{2}\right)\right)=0$. If $x_{1}\left(t_{1}\right)=$ $x_{1}\left(t_{2}\right)$ holds, (3.3) is clearly true. Now assume $x_{1}\left(t_{1}\right)<x_{1}\left(t_{2}\right)$.

Set $F(x) \triangleq g(x)+h(x)$, according to $\left(\mathrm{H}_{1}\right)$, one has

$$
\begin{aligned}
F\left(x_{1}\left(t_{1}\right)\right) & =g\left(x_{1}\left(t_{1}\right)\right)+h\left(x_{1}\left(t_{1}\right)\right) \\
& <g\left(x_{1}\left(t_{1}\right)\right)+h\left(x_{1}\left(t_{2}\right)\right) \\
& <g\left(x_{1}\left(t_{2}\right)\right)+h\left(x_{1}\left(t_{2}\right)\right)=F\left(x_{1}\left(t_{2}\right)\right),
\end{aligned}
$$

namely $F\left(x_{1}\left(t_{1}\right)\right)<0<F\left(x_{1}\left(t_{2}\right)\right)$, then there must exist a $t_{0}$ such that $F\left(x_{1}\left(t_{0}\right)\right)=0$. That is, $g\left(x_{1}\left(t_{0}\right)\right)+h\left(x_{1}\left(t_{0}\right)\right)=0$. According to $\left(\mathrm{H}_{2}\right),(3.3)$ holds. If $x_{1}\left(t_{1}\right)>x_{1}\left(t_{2}\right)$ holds, by a similar method, we can show that (3.3) is also true.

Let $\tilde{t}=n T+t_{0}$, where $\tilde{t} \in[0, T]$ and $n$ is an integer. Then

$$
\left|x_{1}(t)\right|=\left|x_{1}(\tilde{t})+\int_{\tilde{t}}^{t} x_{1}^{\prime}(s) d s\right| \leq d+\int_{0}^{T}\left|x_{1}^{\prime}(s)\right| d s \quad \text { for all } s \in[0, T] \text {. }
$$

Thus we get

$$
\left|x_{1}\right|_{0}=\max _{t \in[0, T]}\left|x_{1}(t)\right| \leq d+\sqrt{T}\left|x_{1}^{\prime}\right|_{2} .
$$

On the other hand, multiplying both sides of the second equation of (3.1) by $x_{1}^{\prime}(t)$ and integrating over $[0, T]$, we have

$$
\begin{aligned}
& \int_{0}^{T}\left(\phi^{-1}\left(\frac{x_{1}^{\prime}(t)}{\lambda}\right)\right)^{\prime} x_{1}^{\prime}(t) d t+\int_{0}^{T} f\left(x_{1}(t)\right)\left(x_{1}^{\prime}(t)\right)^{2} d t \\
& \quad+\lambda \int_{0}^{T} g\left(x_{1}(t-\tau(t))\right) x_{1}^{\prime}(t) d t+\lambda \int_{0}^{T} h\left(x_{1}(t-\gamma(t))\right) x_{1}^{\prime}(t) d t \\
& =\lambda \int_{0}^{T} e(t) x_{1}^{\prime}(t) d t,
\end{aligned}
$$


where $\phi^{-1}\left(\frac{x_{1}^{\prime}(t)}{\lambda}\right)=\varphi_{p}\left(\frac{\frac{x_{1}^{\prime}(t)}{\lambda}}{\sqrt{1+\left(\frac{x_{1}^{\prime}(t)}{\lambda}\right)^{2}}}\right)$. Furthermore, in view of $\left(\mathrm{H}_{4}\right)$, we have

$$
\begin{aligned}
& \left|\int_{0}^{T} f\left(x_{1}(t)\right)\left(x_{1}^{\prime}(t)\right)^{2} d t\right| \\
& \quad=\int_{0}^{T}\left|f\left(x_{1}(t)\right)\right|\left|x_{1}^{\prime}(t)\right|^{2} d t \\
& \quad \leq\left|\int_{0}^{T}\left(\phi^{-1}\left(\frac{x_{1}^{\prime}(t)}{\lambda}\right)\right)^{\prime} x_{1}^{\prime}(t) d t\right|+\lambda\left|\int_{0}^{T} g\left(x_{1}(t-\tau(t))\right) x_{1}^{\prime}(t) d t\right| \\
& \quad+\lambda\left|\int_{0}^{T} h\left(x_{1}(t-\gamma(t))\right) x_{1}^{\prime}(t) d t\right|+\lambda\left|\int_{0}^{T} e(t) x_{1}^{\prime}(t) d t\right| .
\end{aligned}
$$

Denote $w(t)=\phi^{-1}\left(x_{1}^{\prime}(t) / \lambda\right)$, then

$$
\int_{0}^{T}\left(\phi^{-1}\left(x_{1}^{\prime}(t) / \lambda\right)\right)^{\prime} x_{1}^{\prime}(t) d t=\lambda \int_{0}^{T} \phi(\omega(t)) d \omega(t)=0
$$

Together with (3.6) and the fact $\lambda \in(0,1)$ yields

$$
\begin{aligned}
\sigma \int_{0}^{T}\left|x_{1}^{\prime}(t)\right|^{2} d t \leq & \left|\int_{0}^{T} g\left(x_{1}(t-\tau(t))\right) x_{1}^{\prime}(t) d t\right|+\left|\int_{0}^{T} h\left(x_{1}(t-\gamma(t))\right) x_{1}^{\prime}(t) d t\right| \\
& +\left|\int_{0}^{T} e(t) x_{1}^{\prime}(t) d t\right| .
\end{aligned}
$$

Set

$$
\begin{array}{ll}
E_{1}=\left\{t \in[0,1],\left|x_{1}(t-\tau(t))\right| \leq d\right\}, & E_{2}=\left\{t \in[0,1],\left|x_{1}(t-\tau(t))\right|>d\right\}, \\
E_{3}=\left\{t \in[0,1],\left|x_{1}(t-\gamma(t))\right| \leq d\right\}, & E_{4}=\left\{t \in[0,1],\left|x_{1}(t-\gamma(t))\right|>d\right\} .
\end{array}
$$

In view of Lemma 2.3, we obtain

$$
\begin{aligned}
\sigma\left|x_{1}^{\prime}\right|_{2}^{2} \leq & \int_{E_{1}}\left|g\left(x_{1}(t-\tau(t))\right)\right|\left|x_{1}^{\prime}(t)\right| d t+\int_{E_{2}}\left|g\left(x_{1}(t-\tau(t))\right)\right|\left|x_{1}^{\prime}(t)\right| d t \\
& +\int_{E_{3}}\left|h\left(x_{1}(t-\gamma(t))\right)\right|\left|x_{1}^{\prime}(t)\right| d t+\int_{E_{4}}\left|h\left(x_{1}(t-\gamma(t))\right)\right|\left|x_{1}^{\prime}(t)\right| d t \\
& +\int_{0}^{T}|e(t)|\left|x_{1}^{\prime}(t)\right| d t \\
\leq & g_{d} \int_{0}^{T}\left|x_{1}^{\prime}(t)\right| d t+h_{d} \int_{0}^{T}\left|x_{1}^{\prime}(t)\right| d t+k_{1} \int_{0}^{T}\left|x_{1}(t-\tau(t))\right|\left|x_{1}^{\prime}(t)\right| d t \\
& +k_{2} \int_{0}^{T}\left|x_{1}(t-\gamma(t))\right|\left|x_{1}^{\prime}(t)\right| d t+c_{1} \int_{0}^{T}\left|x_{1}^{\prime}(t)\right| d t+c_{2} \int_{0}^{T}\left|x_{1}^{\prime}(t)\right| d t \\
& +|e|_{\infty} \int_{0}^{T}\left|x_{1}^{\prime}(t)\right| d t \\
\leq & \left(g_{d}+h_{d}+c_{1}+c_{2}+|e|_{\infty}\right) \sqrt{T}\left|x_{1}^{\prime}\right|_{2}+k_{1} \sqrt{\tau_{0}}\left|x_{1}^{\prime}\right|_{2}\left|x_{1}\right|_{2} \\
& +k_{2} \sqrt{\gamma_{0}}\left|x_{1}^{\prime}\right|_{2}\left|x_{1}\right|_{2},
\end{aligned}
$$


where $\tau_{0}, \gamma_{0}$ are defined by Lemma 2.3 , and

$$
g_{d}=\max _{|x| \leq d}|g(x)|, \quad h_{d}=\max _{|x| \leq d}|h(x)|, \quad|e|_{\infty}=\max _{t \in[0, T]}|e(t)| .
$$

From (3.3), there exists a point $t_{0} \in R$ such that $\left|x_{1}\left(t_{0}\right)\right| \leq d$. Let $\varpi(t)=x_{1}(t)-x_{1}\left(t_{0}\right)$, we have $\varpi(t+T)=\varpi(t), \varpi^{\prime}(t)=x_{1}^{\prime}(t)$, and $\varpi\left(t_{0}\right)=0$; then, by Lemma 2.2,

$$
|\varpi|_{2} \leq \frac{T}{\pi}\left|\varpi^{\prime}\right|_{2}=\frac{T}{\pi}\left|x_{1}^{\prime}\right|_{2} .
$$

By the Minkowski inequality, we get

$$
\begin{aligned}
\left|x_{1}\right|_{2} & =\left(\int_{0}^{T}\left|x_{1}(t)\right|^{2} d t\right)^{\frac{1}{2}}=\left(\int_{0}^{T}\left|\varpi(t)+x_{1}\left(t_{0}\right)\right|^{2} d t\right)^{\frac{1}{2}} \\
& \leq\left(\int_{0}^{T}\left|x_{1}\left(t_{0}\right)\right|^{2} d t\right)^{\frac{1}{2}}+\left(\int_{0}^{T}|\varpi(t)|^{2} d t\right)^{\frac{1}{2}} .
\end{aligned}
$$

So

$$
\left|x_{1}\right|_{2} \leq \sqrt{T} d+|\varpi|_{2} \leq \sqrt{T} d+\frac{T}{\pi}\left|x_{1}^{\prime}\right|_{2} .
$$

Combining (3.7) and (3.8), we obtain

$$
\begin{aligned}
\sigma\left|x_{1}^{\prime}\right|_{2}^{2} \leq & \left(g_{d}+h_{d}+c_{1}+c_{2}+|e|_{\infty}\right) \sqrt{T}\left|x_{1}^{\prime}\right|_{2}+\left(k_{1} \sqrt{\tau_{0}}+k_{2} \sqrt{\gamma_{0}}\right)\left|x_{1}^{\prime}\right|_{2}\left(\sqrt{T} d+\frac{T}{\pi}\left|x_{1}^{\prime}\right|_{2}\right) \\
= & \left(g_{d}+h_{d}+c_{1}+c_{2}+|e|_{\infty}\right) \sqrt{T}\left|x_{1}^{\prime}\right|_{2}+\left(k_{1} \sqrt{\tau_{0}}+k_{2} \sqrt{\gamma_{0}}\right) \sqrt{T} d\left|x_{1}^{\prime}\right|_{2} \\
& +\frac{T}{\pi}\left(k_{1} \sqrt{\tau_{0}}+k_{2} \sqrt{\gamma_{0}}\right)\left|x_{1}^{\prime}\right|_{2}^{2} .
\end{aligned}
$$

Since $\frac{T}{\pi}\left(k_{1} \sqrt{\tau_{0}}+k_{2} \sqrt{\gamma_{0}}\right)<\sigma$, there is a constant $M_{0}>0$ independent of $\lambda$ such that $\left|x_{1}^{\prime}\right|_{2} \leq$ $M_{0}$, i.e., $\left|x_{1}\right|_{0} \leq d+\sqrt{T}\left|x_{1}^{\prime}\right|_{2} \leq d+\sqrt{T} M_{0}:=M_{1}$.

By the first equation of (3.1), we have

$$
\int_{0}^{T} \lambda \frac{\varphi_{q}\left(x_{2}(t)\right)}{\sqrt{1-\varphi_{q}^{2}\left(x_{2}(t)\right)}} d t=0,
$$

together with $\varphi_{q}(0)=0$, which implies that there is a constant $\eta \in[0, T]$ such that $x_{2}(\eta)=0$. Hence

$$
\left|x_{2}\right|_{0} \leq \int_{0}^{\eta}\left|x_{2}^{\prime}(s)\right| d s \leq \int_{0}^{T}\left|x_{2}^{\prime}(s)\right| d s .
$$

By the two equations of (3.1) and Hölder's inequality, we obtain

$$
\begin{aligned}
\int_{0}^{T}\left|x_{2}^{\prime}(s)\right| d s \leq & \int_{0}^{T}\left|f\left(x_{1}(t)\right) x_{1}^{\prime}(t)\right| d t+\lambda \int_{0}^{T}\left|g\left(x_{1}(t-\tau(t))\right)\right| d t \\
& +\lambda \int_{0}^{T}\left|h\left(x_{1}(t-\gamma(t))\right)\right| d t+\lambda \int_{0}^{T}|e(t)| d t
\end{aligned}
$$




$$
\begin{aligned}
& \leq \int_{0}^{T}\left|f\left(x_{1}(t)\right)\right|\left|x_{1}^{\prime}(t)\right| d t+g_{M_{1}} T+h_{M_{1}} T+|e|_{\infty} T \\
& <|f|_{0} M_{0} \sqrt{T}+\left(g_{M_{1}}+h_{M_{1}}+|e|_{\infty}\right) T
\end{aligned}
$$

where $|f|_{0}=\max _{\left|x_{1}\right| \leq M_{1}}\left|f\left(x_{1}\right)\right|, g_{M_{1}}=\max _{\left|x_{1}\right| \leq M_{1}}\left|g\left(x_{1}\right)\right|, h_{M_{1}}=\max _{\left|x_{1}\right| \leq M_{1}}\left|h\left(x_{1}\right)\right|$. By (3.10), (3.11), $\left|x_{2}\right|_{0} \leq\left(g_{M_{1}}+h_{M_{1}}+|e|_{\infty}\right) T+|f|_{0} M_{0} \sqrt{T}+d=: M_{2}$.

Let $\Omega_{2}=\{x \in \operatorname{Ker} L: N x \in \operatorname{Im} L\}$. If $x \in \Omega_{2}$, then $Q N x=0$. In view of $\int_{0}^{T} e(t) d t=0$, we have

$$
\left\{\begin{array}{l}
\frac{\varphi_{q}\left(x_{2}\right)}{\sqrt{1-\varphi_{q}^{2}\left(x_{2}\right)}}=0 \\
\int_{0}^{T} g\left(x_{1}\right) d t+\int_{0}^{T} h\left(x_{1}\right) d t=0
\end{array}\right.
$$

So $x_{2}=0 \leq M_{2}$. Together with $\left(\mathrm{H}_{2}\right)$ yields

$$
\left|x_{1}\right| \leq d \leq M_{2}
$$

Let $\Omega=\left\{x: x=\left(x_{1}, x_{2}\right) \in X,\left|x_{1}\right|_{0}<M_{1}+1,\left|x_{2}\right|_{0}<M_{2}+1\right\}$. Then $\Omega \supset\left(\Omega_{1} \cup \Omega_{2}\right)$ and $\Omega$ is a bounded open set of $X$. So (1) and (2) of Lemma 2.1 are satisfied.

In the next step we show that condition (3) of Lemma 2.1 holds. Define a linear isomorphism $J: \operatorname{Im} Q \rightarrow \operatorname{Ker} L$ by $J\left(x_{1}, x_{2}\right)=\left(-x_{2}, x_{1}\right)$, and let

$$
H(v, \mu):=\mu v+\frac{1-\mu}{T} J Q N v, \quad(v, \mu) \in \Omega \times[0,1] .
$$

The direct computation and $\left(\mathrm{H}_{2}\right)$ show that for $(x, \mu) \in \partial(\Omega \cap \operatorname{Ker} L) \times[0,1]$,

$$
x^{\top} H(x, \mu)=\mu\left(x_{1}^{2}+x_{2}^{2}\right)+\frac{1-\mu}{T}\left(g\left(x_{1}\right)+h\left(x_{1}\right)\right) x_{1}+\frac{1-\mu}{T} \frac{\left|x_{2}\right|^{q-2} x_{2}^{2}}{\sqrt{1-\varphi_{q}^{2}\left(x_{2}\right)}}>0 .
$$

Thus, $x^{\top} H(x, \mu) \neq 0$ for $(x, \mu) \in \partial \Omega \cap \operatorname{Ker} L \times[0,1]$, which implies

$$
\begin{aligned}
\operatorname{deg}\{J Q N, \Omega \cap \operatorname{Ker} L, 0\} & =\operatorname{deg}\{H(x, 0), \Omega \cap \operatorname{Ker} L, 0\}=\operatorname{deg}\{H(x, 1), \Omega \cap \operatorname{Ker} L, 0\} \\
& =\operatorname{deg}\{I, \Omega \cap \operatorname{Ker} L, 0\} \neq 0 .
\end{aligned}
$$

Condition (3) of Lemma 2.1 holds. By Lemma 2.1, the equation $L x=N x$ has a solution. This completes the proof of Theorem 3.1.

We can use a similar method to conclude the following result, the details are omitted.

Theorem 3.2 Suppose $\left(\mathrm{H}_{3}\right),\left(\mathrm{H}_{4}\right)$, and the following assumptions hold:

$\left(\mathrm{H}_{1}^{\prime}\right)\left(g\left(x_{1}\right)-g\left(x_{2}\right)\right)\left(x_{1}-x_{2}\right)<0, x_{1}, x_{2} \in R$, and $x_{1} \neq x_{2},\left(h\left(x_{1}\right)-h\left(x_{2}\right)\right)\left(x_{1}-x_{2}\right)<0, x_{1}, x_{2} \in R$, and $x_{1} \neq x_{2}$.

$\left(\mathrm{H}_{2}^{\prime}\right)$ There is a constant $d>0$ such that $x(g(x)+h(x))<0$ if and only if $|x|>d$.

Then (1.3) has at least one T-periodic solution if $\frac{T}{\pi}\left(k_{1} \sqrt{\tau_{0}}+k_{2} \sqrt{\gamma_{0}}\right)<\sigma$.

\section{Uniqueness results}

It is difficult to establish the uniqueness of the $T$-periodic solution of (1.3), but in the special case we obtain the uniqueness of (1.3). 
Theorem 4.1 Assume $\left(\mathrm{H}_{1}^{\prime}\right)$ holds, and $\tau(t)=\varepsilon_{1}, \gamma(t)=\varepsilon_{2}\left(\varepsilon_{1}, \varepsilon_{2}\right.$ are sufficiently small constants). Then (1.3) has at most one T-periodic solution.

Proof Denote

$$
I(x)=\int_{0}^{x} f(w) d w, \quad y(t)=\varphi_{p}\left(\frac{x^{\prime}(t)}{\sqrt{1+x^{\prime 2}(t)}}\right)+I(x(t)) .
$$

Then (1.3) can be converted into the following form:

$$
\left\{\begin{array}{l}
x^{\prime}(t)=\frac{\varphi_{q}(y(t)-I(x(t)))}{\sqrt{1-\varphi_{q}^{2}(y(t)-I(x(t)))}} \\
y^{\prime}(t)=-g\left(x\left(t-\varepsilon_{1}\right)\right)-h\left(x\left(t-\varepsilon_{2}\right)\right)+e(t) .
\end{array}\right.
$$

Suppose that $x_{i}(t)(i=1,2)$ are two $T$-periodic solutions of (1.3). Then from (4.1), we have

$$
\left\{\begin{array}{l}
x_{i}^{\prime}(t)=\frac{\varphi_{q}\left(y_{i}(t)-I\left(x_{i}(t)\right)\right)}{\sqrt{1-\varphi_{q}^{2}\left(y_{i}(t)-I\left(x_{i}(t)\right)\right)}}, \\
y_{i}^{\prime}(t)=-g\left(x_{i}\left(t-\varepsilon_{1}\right)\right)-h\left(x_{i}\left(t-\varepsilon_{2}\right)\right)+e(t), \quad i=1,2 .
\end{array}\right.
$$

Let $u(t)=x_{1}(t)-x_{2}(t), v(t)=y_{1}(t)-y_{2}(t)$, which together with (4.2) yields

$$
\left\{\begin{aligned}
u^{\prime}(t)= & \frac{\varphi_{q}\left(y_{1}(t)-I\left(x_{1}(t)\right)\right)}{\sqrt{1-\varphi_{q}^{2}\left(v_{1}(t)-I\left(x_{1}(t)\right)\right)}}-\frac{\varphi_{q}\left(\left(_{2}(t)-I\left(x_{2}(t)\right)\right)\right.}{\sqrt{1-\varphi_{q}^{2}\left(y_{2}(t)-I\left(x_{2}(t)\right)\right)}}, \\
v^{\prime}(t)= & -\left(g\left(x_{1}\left(t-\varepsilon_{1}\right)\right)-g\left(x_{2}\left(t-\varepsilon_{1}\right)\right)\right) \\
& -\left(h\left(x_{1}\left(t-\varepsilon_{2}\right)\right)-h\left(x_{2}\left(t-\varepsilon_{2}\right)\right)\right) .
\end{aligned}\right.
$$

Now, we claim that

$$
v(t) \leq 0, \quad \forall t \in R
$$

We argue by contradiction, in view of $v \in C^{2}[0, T]$ and $v(t+T)=v(t), \forall t \in R$, we obtain $\max _{t \in R} v(t)>0$.

Then there must exist $t^{*} \in R$ such that

$$
\left\{\begin{aligned}
v\left(t^{*}\right)= & \max _{t \in[0, T]} v(t)=\max _{t \in R} v(t)>0 \\
v^{\prime}\left(t^{*}\right)= & -\left(g\left(x_{1}\left(t^{*}-\varepsilon_{1}\right)\right)-g\left(x_{2}\left(t^{*}-\varepsilon_{1}\right)\right)\right) \\
& -\left(h\left(x_{1}\left(t^{*}-\varepsilon_{2}\right)\right)-h\left(x_{2}\left(t^{*}-\varepsilon_{2}\right)\right)\right)=0 \\
v^{\prime \prime}\left(t^{*}\right)= & -\left(g^{\prime}\left(x_{1}\left(t^{*}-\varepsilon_{1}\right)\right) x_{1}^{\prime}\left(t^{*}-\varepsilon_{1}\right)-g^{\prime}\left(x_{2}\left(t^{*}-\varepsilon_{1}\right)\right) x_{2}^{\prime}\left(t^{*}-\varepsilon_{1}\right)\right) \\
& -\left(h^{\prime}\left(x_{1}\left(t^{*}-\varepsilon_{2}\right)\right) x_{1}^{\prime}\left(t^{*}-\varepsilon_{2}\right)-h^{\prime}\left(x_{2}\left(t^{*}-\varepsilon_{2}\right)\right) x_{2}^{\prime}\left(t^{*}-\varepsilon_{2}\right)\right) \leq 0 .
\end{aligned}\right.
$$

Noticing $\left(\mathrm{H}_{1}^{\prime}\right)$, that is, $g^{\prime}(x)<0, h^{\prime}(x)<0, \varepsilon_{1}, \varepsilon_{2}$ sufficiently small, it follows from the second equation of (4.4) that

$$
x_{1}\left(t^{*}-\varepsilon_{1}\right) \equiv x_{2}\left(t^{*}-\varepsilon_{1}\right), \quad x_{1}\left(t^{*}-\varepsilon_{2}\right) \equiv x_{2}\left(t^{*}-\varepsilon_{2}\right) .
$$

Then from the third equation of (4.4), we get

$$
\begin{aligned}
v^{\prime \prime}\left(t^{*}\right)= & -g^{\prime}\left(x_{1}\left(t^{*}-\varepsilon_{1}\right)\right)\left(x_{1}^{\prime}\left(t^{*}-\varepsilon_{1}\right)-x_{2}^{\prime}\left(t^{*}-\varepsilon_{1}\right)\right) \\
& -h^{\prime}\left(x_{1}\left(t^{*}-\varepsilon_{2}\right)\right)\left(x_{1}^{\prime}\left(t^{*}-\varepsilon_{2}\right)-x_{2}^{\prime}\left(t^{*}-\varepsilon_{2}\right)\right)
\end{aligned}
$$




$$
\begin{aligned}
= & -g^{\prime}\left(x_{1}\left(t^{*}-\varepsilon_{1}\right)\right)\left(\frac{\varphi_{q}\left(y_{1}\left(t^{*}-\varepsilon_{1}\right)-I\left(x_{1}\left(t^{*}-\varepsilon_{1}\right)\right)\right)}{\sqrt{1-\varphi_{q}^{2}\left(y_{1}\left(t^{*}-\varepsilon_{1}\right)-I\left(x_{1}\left(t^{*}-\varepsilon_{1}\right)\right)\right)}}\right. \\
& \left.-\frac{\varphi_{q}\left(y_{2}\left(t^{*}-\varepsilon_{1}\right)-I\left(x_{2}\left(t^{*}-\varepsilon_{1}\right)\right)\right)}{\sqrt{1-\varphi_{q}^{2}\left(y_{2}\left(t^{*}-\varepsilon_{1}\right)-I\left(x_{2}\left(t^{*}-\varepsilon_{1}\right)\right)\right)}}\right) \\
& -h^{\prime}\left(x_{1}\left(t^{*}-\varepsilon_{2}\right)\right)\left(\frac{\varphi_{q}\left(y_{1}\left(t^{*}-\varepsilon_{2}\right)-I\left(x_{1}\left(t^{*}-\varepsilon_{1}\right)\right)\right)}{\sqrt{1-\varphi_{q}^{2}\left(y_{1}\left(t^{*}-\varepsilon_{2}\right)-I\left(x_{1}\left(t^{*}-\varepsilon_{2}\right)\right)\right)}}\right. \\
& \left.-\frac{\varphi_{q}\left(y_{2}\left(t^{*}-\varepsilon_{2}\right)-I\left(x_{2}\left(t^{*}-\varepsilon_{2}\right)\right)\right)}{\sqrt{1-\varphi_{q}^{2}\left(y_{2}\left(t^{*}-\varepsilon_{2}\right)-I\left(x_{2}\left(t^{*}-\varepsilon_{2}\right)\right)\right)}}\right) .
\end{aligned}
$$

In view of $g^{\prime}\left(x_{1}\left(t^{*}-\varepsilon_{1}\right)\right)<0, h^{\prime}\left(x_{1}\left(t^{*}-\varepsilon_{2}\right)\right)<0, v\left(t^{*}\right)=y_{1}\left(t^{*}\right)-y_{2}\left(t^{*}\right)>0, \frac{x}{\sqrt{1-x^{2}}}, \varphi_{q}(x)$ are increasing on $x$, and $\varepsilon_{1}, \varepsilon_{2}$ are sufficiently small, thus we have

$$
v^{\prime \prime}\left(t^{*}\right)>0 .
$$

This contradicts the third equation of (4.4), which implies that

$$
v(t)=y_{1}(t)-y_{2}(t) \leq 0, \quad \forall t \in R
$$

By using a similar argument, we can also show that $y_{2}(t)-y_{1}(t) \leq 0$.

Hence, we obtain

$$
y_{1}(t) \equiv y_{2}(t), \quad \forall t \in R
$$

Then from (4.3) and $g^{\prime}(x)<0$, we have $x_{1}\left(t-\varepsilon_{1}\right) \equiv x_{2}\left(t-\varepsilon_{1}\right), x_{1}\left(t-\varepsilon_{2}\right) \equiv x_{2}\left(t-\varepsilon_{2}\right), \forall t \in R$. That is

$$
x_{1}(t) \equiv x_{2}(t)
$$

Therefore, (1.3) has at most one $T$-periodic solution. The proof of Theorem 4.1 is now complete.

From Theorem 3.2 and Theorem 4.1, we see that (1.3) has an unique $T$-periodic solution when $\tau(t)=\varepsilon_{1}, \gamma(t)=\varepsilon_{2}$.

\section{An example}

In this section we give an example to illustrate the application of Theorem 3.1.

Example 5.1 Consider the prescribed mean curvature Liénard equation with two deviating arguments:

$$
\begin{aligned}
& \left(\varphi_{4}\left(\frac{x^{\prime}(t)}{\sqrt{1+x^{\prime 2}(t)}}\right)\right)^{\prime}+f(x(t)) x^{\prime}(t)+g\left(x\left(t-\frac{\cos 3 \pi t}{4 \pi}\right)\right)+h\left(x\left(t-\frac{\sin 3 \pi t}{4 \pi}\right)\right) \\
& =\sin 3 \pi t,
\end{aligned}
$$


where $f(x)=\sigma+x^{2}, \sigma>5, \tau(t)=\frac{\cos 3 \pi t}{4 \pi}, \gamma(t)=\frac{\sin 3 \pi t}{4 \pi}, e(t)=\sin 3 \pi t, g(x)=\frac{85}{16} x+6, h(x)=$ $5 x+2$. So $\tau_{0}=\gamma_{0}=4$. Choosing $T=\frac{2}{3}, d=10, k_{1}=\frac{85}{16}, k_{2}=5$, then $\left(k_{1} \sqrt{\tau_{0}}+k_{2} \sqrt{\gamma_{0}}\right) \frac{T}{\pi} \approx$ $4.38<5<\sigma$. It is obvious that $\left(\mathrm{H}_{1}\right),\left(\mathrm{H}_{2}\right),\left(\mathrm{H}_{3}\right)$, and $\left(\mathrm{H}_{4}\right)$ hold. Then it follows from Theorem 3.1 that (5.1) has at least one $\frac{2}{3}$-periodic solution.

\section{Competing interests}

The authors declare that they have no competing interests.

\section{Authors' contributions}

All authors have equally contributed in obtaining new results in this article and also read and approved the final manuscript.

\section{Author details}

'College of Science, Hohai University, Nanjing, 210098, China. ${ }^{2}$ Department of Mathematics and Physics, Hohai University, Changzhou Campus, Changzhou, 213022, China. ${ }^{3}$ Department of Mathematics, Beijing Institute of Technology, Beijing, 100081, China.

\section{Acknowledgements}

This work was sponsored by the NSFC (11471073) and the Fundamental Research Funds for the Central Universities (2014B11414). The authors are very grateful to the referee for his/her careful reading of the manuscript and for his/her valuable suggestions on improving this article.

\section{Received: 2 September 2014 Accepted: 13 November 2014 Published: 25 Nov 2014}

\section{References}

1. Li, W, Liu, Z: Exact number of solutions of a prescribed mean curvature equation. J. Math. Anal. Appl. 367, 486-498 (2010)

2. Zhang, X, Feng, M: Exact number of solutions of a one-dimensional prescribed mean curvature equation with concave-convex nonlinearities. J. Math. Anal. Appl. 395, 393-402 (2012)

3. Pan, $\mathrm{H}$, Xing, $\mathrm{R}$ : Time maps and exact multiplicity results for one-dimensional prescribed mean curvature equations $\mathrm{II}$. Nonlinear Anal. TMA 74, 3751-3768 (2011)

4. Feng, M: Periodic solutions for prescribed mean curvature Liénard equation with a deviating argument. Nonlinear Anal., Real World Appl. 13, 1216-1223 (2012)

5. Lu, S, Lu, M: Periodic solutions for a prescribed mean curvature equation with multiple delays. J. Appl. Math. 2014 Article ID 909252 (2014). doi:10.1155/2014/909252

6. Cheung, W, Ren, J: Periodic solutions for $p$-Laplacian Liénard equation with a deviating argument. Nonlinear Anal. 59 . 107-120 (2004)

7. Cheung, W, Ren, J: On the existence of periodic solutions for $p$-Laplacian generalized Liénard equation. Nonlinear Anal. 60, 65-75 (2005)

8. Lu, S, Ge, W: Periodic solutions for a kind of Liénard equations with deviating arguments. J. Math. Anal. Appl. 249, 231-243 (2004)

9. Wang, D: Existence and uniqueness of periodic solution for prescribed mean curvature Rayleigh type $p$-Laplacian equation. J. Appl. Math. Comput. 46, 181-200 (2014)

10. Gaines, RE, Mawhin, J: Coincidence Degree and Nonlinear Differential Equations. Lecture Notes in Mathematics, vol. 568. Springer, Berlin (1977)

11. Liu, X, Jia, M, Ren, R: On the existence and uniqueness of periodic solutions to a type of Duffing equation with complex arguments. Acta Math. Sci. Ser. A 27, 37-44 (2007) (in Chinese)

12. Wang, Z, Qian, L, Lu, S, Cao, J: The existence and uniqueness of periodic solutions for a kind of Duffing-type equation with two deviating arguments. Nonlinear Anal. 73, 3034-3043 (2010)

10.1186/1687-1847-2014-290

Cite this article as: Li et al.: Existence of periodic solutions for a prescribed mean curvature Liénard $p$-Laplacian equation with two delays. Advances in Difference Equations 2014, 2014:290 